\title{
Integer Programming Formulations for the Uncapacitated Vehicle Routing p-Hub Center Problem
}

\author{
Z. Kartal $^{\mathrm{a}}{ }^{\text {and A.T. Ernst }}{ }^{\mathrm{b}}$ \\ ${ }^{a}$ Anadolu University, Department of Industrial Engineering, 26555, Eskisehir, Turkey \\ ${ }^{\mathrm{b}}$ CSIRO, Private Bag 33, Clayton South, Vic. 3169, Australia \\ Email: zkartal@anadolu.edu.tr
}

\begin{abstract}
Hub facilities are used in many-to-many transportation networks such as passenger airlines, parcel delivery, and telecommunication system networks. In these networks, the flow that is interchanged between the demand centers is routed via the hubs to provide discounted transport. Many parcel delivery firms serve on a hub based system where the flows from different demand nodes are concentrated, sorted and disseminated at the hub centers in order to transfer them to the destinations points. The main purpose of hub location problem is to decide the location of hub facilities and to allocate demand nodes to the hubs. In hub based systems, as an alternative way of serving each origin destination node directly, the flow is accumulated at the hub facilities in order to exploit the substantial economies of scale.
\end{abstract}

Hub location problems can be categorized in terms of the objective function of the mathematical models. In the literature, hub location problems with total transportation cost objectives (median), min-max type objectives (center) and covering type objectives are well studied.

In the hub location problem literature, it is assumed that only one vehicle serves between each demand center and hub. The vehicles are not permitted to visit more than one city. The need to design a network of combined hub locations and vehicle routes arises in various applications. For example, in cargo delivery systems, sending separate vehicles between each demand center and hub is rather costly in terms of investment on the total number of vehicles. Instead, if the vehicles are allowed to follow a route by visiting different demand nodes in each stop, the total investment cost may decrease considerably. In airline companies, similarly, if a separate aircraft and separate air staff are assigned for each destination, they incur high investment and operating costs. Also, traffic congestion occurs at airports and in air networks.

In the light of above-mentioned real life considerations, the vehicle routing hub location problem has been receiving increased attention from researchers. This problem is to decide the location of hubs, the allocation demand centers to the hubs and the associated routing structure with multiple stopovers and allowing vehicles to make a tour so as to minimize total transportation cost.

In addition to the cost, parallel to the increase of the competition in the market, companies tend to promise to the customers 'next day delivery' or 'delivery within $24 \mathrm{~h}$ ' guarantees. However, the hub location and vehicle routing problem, which consider both the flows and distances, may sometimes lead to delays from non-simultaneous arrivals at hubs, when worst-case route lengths for vehicles are excessively large. Although classical hub location problems provide one option when origin-destination distances are huge, they become less appropriate when vehicle routing is required and delivery time is a major concern.

In this study, we introduce the uncapacitated vehicle routing $p$-hub center problem to the literature. The aim of our model is to find the location of the hubs, assign demand centers to the hubs and determine the routes of vehicles for each hub such that the maximum distance or travel time between origin-destination pairs is minimized. We propose mathematical programming formulations for this problem with $\mathrm{O}\left(n^{2}\right)$ and $\mathrm{O}\left(n^{4}\right)$ variables. The formulations trade off tightness against formulation size. The computational results on standard data sets from the literature allow this trade off to be evaluated empirically and provide an indication of the challenge of solving these combined vehicle routing hub location problems.

Keywords: p-hub center problems, hub location, hub location and routing problem, $p$-hub center and routing problems 


\section{INTRODUCTION}

In most many-to-many distribution systems, goods are collected from various origins to be distributed to various destinations. However, sending the goods directly from each origin to each destination with a dedicated vehicle is often not economical, since the flow rates for a substantial fraction of the origin-destination pairs can sometimes be so small. Hub network systems are used to consolidate the flows between origin-destination pairs to eliminate expensive direct connection.

The aim of the classical single allocation $p$-hub median problem is to minimize the total cost of transportation while establishing $p$ nodes (hubs) among a given set of nodes with pairwise traffic demands and allocate each non-hub node to the hubs. While in the $p$-hub center problem, the main objective is minimizing the maximum distance/cost between origin-destination pairs. In the hub location problems, the flow from $i$ to $j$ must be routed on at least one hub if both the nodes $i$ and $j$ are allocated the same hub; otherwise at most two hubs, if node $i$ and $j$ are allocated to different hubs. There is a path in the route as $i-k-l-j$ if node $i$ is allocated to hub $k$ and node $j$ is allocated to hub $l$. The flow traversing the hub link $k-l$ is the flow from nodes allocated to hub $k$ to nodes allocated to hub $l$. Commonly a discount factor applies on the inter-hub connections since the transportation cost on these links are lower due to economies of scale. Surveys on various different hub location problems can be found in Campbell and O'Kelly [2012] and Alumur and Kara [2008].

The fundamental assumption of hub location problem is that there is a direct connection between each non-hub node and its assigned hub. However, more recently, this unrealistic assumption have been adjusted and adapted to deal with some transportation problems and logistics applications in hub networks where nodes do not have adequate demand to connect them directly with hubs [Rodriguez-Martin et al., 2014].

In this paper, we introduce the uncapacitated vehicle routing $p$-hub center problem (UVRpHCP) to the literature, relaxing the main hub location problem assumption, where there is a separate vehicle which serves between any non-hub node and its assigned hub. To the best of authors' knowledge, no previous study has investigated the combination of $p$-hub center and vehicle routing problems. The aim of the problem is to minimize maximum distance/cost between any origin-destination pair, while locating $p$ hubs, assigning the non-hub nodes to exactly one hub and forming the routing structures of the given vehicles for each hub.

The outline of the paper is as follows. In the next section, we introduce three different mathematical formulations of the UVRpHCP, whose complexities in terms of variables are $\mathrm{O}\left(n^{2}\right)$ and $\mathrm{O}\left(n^{4}\right)$. In the third section, we give some numerical tests with regard to all formulations. Finally, in the last section concluding remarks are presented.

\section{Mathematical Formulations}

The UVRpHCP determines the location of the hubs, the allocation of non-hub nodes to hubs, and the associated routing structure between non-hub nodes and hubs with multiple stopovers so as to minimize maximum distance/cost between origin-destination pairs. This problem can be defined on a complete, symmetric network; $G=(N, A)$ with node set $N=\{1,2, \ldots, n\}$ and $\operatorname{arc}$ set $A$. Each $\operatorname{arc}(i, j) \in N$ has a nonnegative cost (distance), $d_{i j}=d_{j i}$ satisfying the triangle inequality. The economy of scale is modeled by a discount factor $\alpha \in(0,1)$ between hub nodes. The costs can be used to capture travel time, path length or monetary costs.

We assume that a limited fleet of homogenous uncapacitated vehicles $v \in V$ operates on the routes. The number of available vehicles for each hub to deploy; $n(i)$ is known a priori. These vehicles should visit at least two nodes including the hub itself, also they are allowed to visit multiple non-hub nodes. There is no upper bound on the number of nodes that a vehicle can visit. It is also assumed that larger sized and faster vehicles operate between two hubs. It is not permitted for these vehicles make any additional stops along their trips.

One of the main difficulties for solving hub location problems is to deal with larger numbers of variables and constraints in the formulations. Therefore, formulations with fewer variables and constraints have been developed. However, although the larger formulations lead to tighter lower bounds, increased computational times are also required to find and exact solution. For this reason, we first explored an integer linear programming model with $\mathrm{O}\left(n^{4}\right)$ variables to solve UVRpHCP. The motivation behind our first model, which is based on a classical four-index hub location formulation, is to get tighter lower bounds associated with the LP relaxation. To formulate the problem, we use the idea of the Campbell [1994] study, in which $y_{k l}^{i j}$ is defined as the fraction of flow; as being 1 if a vehicle is taking mail originating at node $i$ and destined for node $j$ along the road from node $l$ to node $k$, with $f_{k l}^{i j}$ being analogous for the case where both node $k$ and node $l$ are hubs. We note here 
that while $y_{k l}^{i j}$ and $f_{k l}^{i j}$ variables are allowed to be fractional in the formulation, they are always integer in the optimal solution corresponding to the shortest path from node $i$ and $j$ in the hub network. In this formulation, the path followed by vehicles is defined in the model using the variables $x_{i j}^{v}$ which take on the value 1 if vehicle $v$ visits node $i \in \mathrm{N}$ and node $j \in N \backslash\{i\}$ in a route, and 0 otherwise. In the suggested formulation, there is a three indexed $x_{i j}^{v}$ variable for every arc-vehicle combination.

Let $h_{i}$ to be a zero-one variable with $h_{i}=1$ if node $i \in N$ is a hub and 0 otherwise. Integer variable $z_{i v} \in\{0,1\}$ to denote if vehicle $v \in V$ is assigned to hub $i \in N\left(z_{i v}=1\right)$ or not $\left(z_{i v}=0\right)$.

The mathematical programming formulation of the UVRpHCP with four-indexed flow variables is as follows:

\section{Model-1}

$$
\begin{aligned}
& \min Z_{1} \text {, } \\
& \text { s.t. } \quad \sum_{k} \sum_{l} d_{k l} y_{k l}^{i j}+\sum_{k} \sum_{l} \alpha d_{k l} f_{k l}^{i j} \leq Z_{1}, \quad \forall i, j \\
& f_{k l}^{i j}=f_{l k}^{j i}, \quad \forall i, j \neq i, k, l \neq k, \\
& \sum_{k \neq i}\left(y_{i k}^{i j}+f_{i k}^{i j}\right)=1 \text {, } \\
& \sum_{l \neq k, l \neq i}\left(y_{l k}^{i j}+f_{l k}^{i j}\right)-\sum_{l \neq k, l \neq i}\left(y_{k l}^{i j}+f_{k l}^{i j}\right)=1 \text {, } \\
& \forall i, j \neq i \text {, } \\
& y_{k l}^{i j} \leq \sum_{v} x_{k l}^{v}, \\
& \forall i, j \neq i, k \neq i, k=j, \\
& \forall i, j \neq i, k, l \neq i \\
& \sum_{l}\left(f_{k l}^{i j}+f_{l k}^{i j}\right) \leq h_{k}, \quad \forall i, j, k \in N, \\
& \sum_{i} h_{i}=p \\
& \sum_{j \neq i} x_{i j}^{v}-\sum_{j \neq i} x_{j i}^{v}=0 \\
& \sum_{v} z_{i v}=h_{i} n_{i} \\
& z_{i v} \leq \sum_{j \neq i} x_{i j}^{v} \\
& \sum_{v} \sum_{j \neq i} x_{i j}^{v}=\sum_{v} z_{i v}+\left(1-h_{i}\right), \quad \forall i \\
& \sum_{j \neq i} x_{i j}^{v}-1+h_{i} \leq z_{i v} \\
& \forall i, v \\
& \sum_{i} z_{i v}=1, \\
& z_{i v}, x_{i j}^{v}, h_{i} \in\{0,1\} \\
& 0 \leq f_{k l}^{i j}, y_{k l}^{i j} \leq 1 \\
& \forall i, v \\
& \forall i \\
& \forall i, v \\
& \forall v \\
& \forall i, j \in N, v \in V \\
& \forall i, j, k, l \in N \text {, }
\end{aligned}
$$

The objective function (1) minimizes the maximum distance/cost. The first term in (2) calculates total distance/cost for a route. The second term calculates the total distance between two hubs. Constraint (3) ensures that the fraction of flow that travels from node $i$ to node $j$ via hubs located at $k$ and $l$ is equal to the fraction of flow that follows a route from node $i$ to node $j$ via hubs $l$ and $k$. Constraint (4) guarantees that total flow from $i$ to $j$ is transferred. Constraint (5) is the flow conservation constraint. Constraint (6) defines the amount of vehicle flow originated at node $i$ destined to $j$ uses the link $(j, k)$ from node $j$ to node $k$. Constraint (7) ensures that the total flow is transferred via hub $k$ and hub $l$. Constraint (8) ensures that exactly $p$ hubs are to be located. Constraint (9) is known as degree constraint. With this constraint it is guaranteed that every node has as many vehicles arriving as leaving. If node $i$ is hub, the number of assigned vehicles for this hub is $n(i)$, which is ensured by constraint (10). If node $i$ is hub, the arc between hub node $i$ and non-hub node $j$ is served with exactly one vehicle which is guaranteed by constraint (11). Constraint (12) states that if node $i$ is a hub, the number of arcs that leave from node $i$ is equal to the number of assigned vehicles. On the other 
hand, if node $i$ is not a hub, then this constraint guarantees that each $i-j$ non-hub link is visited only once by a vehicle. By constraint (13), $z_{i v}$ takes value only if node $i$ is a hub node. With constraint (14) it is guaranteed that each vehicle is only allocated to one hub.

This model has $\left(n^{2} v+n v+n\right)$ binary variables, $\left(2 n^{4}\right)$ positive variables and $\left(2 n^{4}+2 n^{3}+2 n^{2}+3 n v+2 n+v\right)$ constraints. Thus, there are $\mathrm{O}\left(n^{4}\right)$ variables and $\mathrm{O}\left(n^{4}\right)$ constraints in total.

Secondly, we explored a three index vehicle flow formulation which uses substantially fewer variables than our $\mathrm{O}\left(n^{4}\right)$ formulation. Therefore, we hope to solve much larger problems with our new formulation which has $\mathrm{O}\left(n^{2}\right)$ variables. Using the same notation as for the four index hub location formulation, we define additional decision variables and parameters;

$a_{i j}: 1$, if node $j$ is the successor node of non-hub node $i$; 0 otherwise.

$b_{i j}^{w}: 1$, if vehicle $w$ travels from node $i$ to node $j ; 0$ otherwise.

$a b_{i j}^{v}: 1$, if vehicle $v$ travels from node $i$ to node $j$, and node $i$ is not a hub; 0 otherwise.

transfer ${ }_{v w}$ : the distance between two hub nodes.

collect $_{v}$ : the longest collection distance from any location served by vehicle $v$ to the hub.

distrib $_{w}$ : the longest distribution distance to any location served by vehicle $w$.

$L:$ the maximum number of nodes a vehicle may visit.

$u_{i}$ : auxiliary variable - defines distance to the hub for subtour elimination.

The second mathematical formulation that we developed for UVRpHCP uses $\mathrm{O}\left(n^{2} v\right)$ variables. Note that since $v$ is typically very small, this formulation is not significantly larger than the third model with $\mathrm{O}\left(n^{2}\right)$ variables.

\section{Model-2}

$\min Z_{2}$

subject to:

$(8)-(15)$

$$
\begin{aligned}
& \sum_{j} a_{i j}=1, \quad \forall i \\
& a_{i j} \leq 1-h_{i}, \quad \forall i, j \\
& a_{i j} \leq \sum_{v} x_{i j}^{v}, \quad \forall i, j \\
& a_{i j} \geq x_{i j}^{v}-h_{i}, \quad \forall i, j, v \\
& u_{i}-u_{j}+L * a_{i j} \leq L-1, \quad \forall i, j \\
& \sum_{j} \sum_{w} b_{i j}^{w}=1, \quad \forall i \\
& b_{i j}^{w} \geq x_{i j}^{w}-h_{j}, \quad \forall i, j, w \\
& \sum_{i} \sum_{j \neq i}\left(b_{i j}^{w} d_{i j}\right)=\text { distrib }_{w}, \quad \forall w \\
& \sum_{j} \sum_{v} a b_{i j}^{v}=1, \quad \forall i \\
& a b_{i j}^{v} \geq x_{i j}^{v}-h_{i}, \quad \forall i, j, v \\
& \sum_{i} \sum_{j \neq i}\left(a b_{i j}^{v} d_{i j}\right)=\text { collect }_{v}, \quad \forall v \\
& \sum_{j} d_{i j} z_{j w}-\max _{j}\left\{d_{i j}\right\}\left(1-z_{i v}\right) \leq \text { transfer }_{v w}, \quad \forall i, v, w \\
& \sum_{i} \sum_{j \neq i} d_{i j} x_{i j}^{v}-d_{k l} x_{k l}^{v}-M\left(1-x_{k l}^{v}\right) \leq \text { collect }_{v}+\text { distrib }_{v}, \quad \forall k, l, v \\
& \text { collect }_{v}+\text { distrib }_{w}+\alpha \times \text { transfer }_{v w} \leq Z_{2}, \quad \forall v \neq w \\
& a_{i j}, b_{i j}^{v}, a b_{i j}^{v} \in\{0,1\} \\
& \forall i, j \in N, v \in V \\
& \text { collect }_{v}, \text { distrib }_{w}, \text { transfer }_{v w}, \max _{i v} \geq 0 \text {, } \\
& \forall i, j \in N, v, w \in V
\end{aligned}
$$


The objective function (17) minimizes the maximum distance/cost. This cost includes the total distance between hubs, plus the distance beginning from the first non-hub node in a vehicle route to the hub (collection) and lastly the distance/cost from the last non-hub node in a vehicle route to the hub (distribution). Since the single allocation scheme is used, each non-hub node $i$ is visited only once which is guaranteed by constraint (18). Constraint (19) ensures that if node $i$ is a hub node, $a_{i j}$ takes on value 0 . On the contrary, if node $i$ is not a hub node, then there should be a link between node $i$ and node $j$. If node $i$ is not a hub node and node $j$ is a hub node, then constraint (20) limits the number of entering arcs into the hub node $j$ to the number of available vehicles. By constraint (21) $a_{i j}$ is one if $i$ is a non-hub node and arc $i-j$ is visited by exactly one vehicle. Constraints (22) are sub-tour elimination constraints. Constraints (23)-(25) together calculate the maximum distribution cost/distance amongst all vehicles. Similarly, constraints (26)-(28) together calculate the maximum collection cost/distance amongst all vehicles. Constraint (29) calculates the maximum cost/distance amongst all hub pairs. When constraint (30) is active the maximum distance is determined by two successive nodes on the route of vehicle $v$, that is we are calculating the distance from $l$ to the hub and then from the hub to node $k$ where arc $(k, l)$ is on the route of $v$. Lastly, constraint (31) ensures that the objective is no less than the travel distance/cost between any pair of nodes allocated to the vehicle $w$ and $v$, since collect $_{v}$ and distrib $_{w}$ give the longest distances of vehicle $v$ and $w$ for different hubs, respectively.

Model-2 for UVRpHCP is a mixed integer program that has $\left(3 n^{2} v+n^{2}+n v+n\right)$ binary variables, $\left(n+v^{2}+2 v\right)$ positive variables and $\left(n^{2} v^{2}+3 n^{2} v+3 n^{2}+n v^{2}+v^{2}+3 n v+5 n+3 v\right)$ constraints. Thus, there are $\mathrm{O}\left(n^{2} v\right)$ variables and $\mathrm{O}\left(n^{2} v^{2}\right)$ constraints in total.

Finally, we describe a third formulation which is based on a two-index vehicle flow formulation that uses $\mathrm{O}$ $\left(n^{2}\right)$ binary variables, that keeps every arc; for the determination path of the vehicle routes. In this formulation, the path followed by vehicles are defined in the model by means of $x_{i j}$ variables which takes on the value 1 if node $i \in N$ precedes node $j \in N \backslash\{i\}$ and 0 otherwise. Integer variables $s_{i v} \in\{0,1\}$ is used to represent if node $i \in N$ is serviced by vehicle $v \in V\left(s_{i v}=1\right)$ or not $\left(s_{i v}=0\right)$. Following the idea of $p$-hub center formulation in Ernst et al. [2009], which is based on the concept of a hub radius, the other variables are $r_{i v}^{1}$ the total distance from the first non-hub node to hub node $i$ in a vehicle route $v$; similarly, $r_{i v}^{2}$ the total distance from the hub node $i$ to last node in a vehicle route $v$. And lastly, in order to calculate the distances of two different vehicles for the same hub, we define $r v_{i v}^{1}$ to be the total distance from the first non-hub node to hub node $i$ in a vehicle route $v ; r v_{i v}^{2}$ denotes the the total distance from the hub node $i$ to last node in a vehicle route $v$. The third formulation which has $\mathrm{O}\left(n^{2}\right)$ binary variables with two index vehicle flow formulation is then given below:

\section{Model-3}

$\min Z_{3}$

subject to:

(8), (14)

$$
\begin{array}{rlrl}
\sum_{j \neq i} x_{i j}-\sum_{j \neq i} x_{j i} & =0, & & \forall i \\
\sum_{j} x_{i j} & =1+\left(n_{i}-1\right) h_{i}, & & \\
\sum_{v} s_{i v} & =1+\left(n_{i}-1\right) h_{i}, & & \\
\sum_{i} s_{i v} \geq 2, & & & \\
z_{i v} & \leq h_{i}, & & \forall v \\
z_{i v} & \leq s_{i v}, & \\
z_{i v} \geq h_{i}+s_{i v}-1, & & \forall i, v \\
s_{i v} \geq s_{j v}+x_{i j}-1-h_{j}, & & \forall i, v \\
s_{i v} & \geq s_{j v}+x_{j i}-1-h_{j}, & & \forall i, v \\
r_{i v}^{1} \geq r_{j v}^{1}+d_{i j}-M\left(1-x_{j i}\right)-M h_{j}-M\left(1-s_{i v}\right), & & \forall i, j, v \\
r_{i v}^{2} \geq r_{j v}^{2}+d_{i j}-M\left(1-x_{i j}\right)-M h_{j}-M\left(1-s_{i v}\right), & & \forall i, j, v \\
r v_{i v}^{1} \geq r v_{j v}^{1}+d_{i j}-M\left(1-x_{i j}\right)-M h_{i}-M\left(1-s_{i v}\right), & \forall i, j, v
\end{array}
$$




$$
\begin{array}{cl}
r v_{i v}^{2} \geq r v_{j v}^{2}+d_{i j}-M\left(1-x_{i j}\right)-M h_{i}-M\left(1-s_{i v}\right), & \forall i, j, v \\
Z_{3} \geq r_{i v}^{1}+\alpha d_{i j}+r_{j w}^{2}-M\left(1-h_{i}\right)-M\left(1-h_{j}\right) & \forall i, j, v, w \\
Z_{3} \geq r v_{i v}^{1}+r v_{j v}^{2}-M\left(1-x_{i j}\right) & \forall i, j, v \\
x_{i j}, s_{i v}, h_{i} \in\{0,1\} & \forall i, j, v \\
r_{i v}^{1}, r_{j v}^{2}, r v_{i v}^{1}, r v_{i v}^{2} \geq 0, & \forall i, j, v,
\end{array}
$$

The objective function (34) minimizes maximum distance between hubs, plus the distance beginning from the first non-hub node in a vehicle route to the hub (collection) and lastly the distance/cost from the last nonhub node in a vehicle route to the hub (distribution). Constraint (35) is known as the degree constraint. By constraints (36) it is guaranteed that if node $i$ is a hub, the number of arcs that leave from node $i$ is equal to the number of assigned vehicles. On the other hand, if node $i$ is not a hub, then these constraints guarantee that each $i-j$ non-hub link is visited only once. Constraint (37) guarantees that each non-hub link is visited by only one vehicle unless node $i$ is not a hub node. Constraint (38) guarantees that for any vehicle the number of visited nodes including hub node itself is greater than or equal to two. With constraint (39) it is guaranteed that each vehicle is only allocated to one hub. By constraint (40) no vehicle is assigned to a node unless a hub is opened at that site. Constraint (41) allows assigning a vehicle to a hub node. Constraints (42) and (43) together guarantee that if there is a link between node $i$ and node $j$, both of these nodes are visited by the same vehicle. Constraint (44) keeps the longest distance for a vehicle from the first non-hub node to hub node in the route (collection) where $\mathrm{M}$ is a large number. Similarly, constraint (45) keeps the longest distance/cost for a vehicle from the hub to the last node of the route (distribution). Constraint (46) calculates the longest collection distance/cost between two nodes in a vehicle route. Constraint (47) calculates the longest distribution distance/cost between two nodes in a vehicle route. If the collection plus distribution cost/distance for two vehicles' of one hub is greater than the two different vehicles of different hubs plus the cost/distance between the hubs, constraint (49) allows that the objective function value must be greater than this value. Notice also here that due to constraints (44), (45), (46) and (47) sub-tours are eliminated. For solving Model-1, $\mathrm{M}$ is taken as the longest distance times number of nodes in the data set.

This mathematical model has $\left(n^{2}+4 n v+n\right)$ binary variables, $(4 n v)$ positive variables and $\left(n^{2} v^{2}+7 n^{2} v+\right.$ $3 n v+3 n+2 v)$ constraints. Thus, there are $\mathrm{O}\left(n^{2}\right)$ variables and constraints in total.

\section{Computational Results}

In this section we present the results with regard to the three formulations that are described in this paper. We solved these using GAMS 23.8 with the GUROBI 4.6 solver on a 64 bit Intel i5 (3.30 Ghz) machine with 8 GB RAM. The effectiveness of our mathematical models were tested on the Civil Aeronautics Board (CAB) data set. This data set, introduced in O'Kelly [1987], is derived from airline passenger data between 25 US cities that includes demands and distances. In order to provide results, we took $n=10$ and $n=15$ nodes problem instances with different numbers of vehicles and hubs. While solving the mathematical models, we limited the CPU time to two hours on GAMS. We report the results from GUROBI performance measures in Table 1.

For each instance in Table 1, the first column gives the characteristics of the test problem n.p. $v$ where $n$ is the number of nodes, $p$ is the number of hubs and $v$ is the number of vehicles that are assigned for each hub. In each instance we set $\alpha=1$. The second column gives the optimal solution. In the instances when any of the mathematical models could not find the optimal solution, reported with an asterisk $\left(^{*}\right)$ in the second column, we give the best integer solution found with one of the mathematical models. The rest of the table has three main parts. We give the same performance measures reported by GUROBI for $\mathrm{O}\left(n^{4}\right)$ variable formulation, $\mathrm{O}$ $\left(n^{2}\right)$ variable with three index vehicle flow formulation, and lastly $\mathrm{O}\left(n^{2}\right)$ variable with two index vehicle flow formulation, referred to as Model-1, Model-2 and Model-3, respectively. In the three columns for three models, we first provide the number of nodes in the branch and bound tree, the CPU time requirement in seconds and gap i.e., $\frac{u b-l b}{u b} * 100$ as reported by GUROBI, where $l b$ is the final lower bound and $u b$ is the final upper bound.

Observe from Table 1 that, Model-2 and Model-3 solved all the instances with 10 nodes optimally in less than one hour of CPU time. Model-2 also solved 15.2.1 to optimality within two hours. We remark here that, the lowest objective values amongst three mathematical models is found by Model-2.

We also observe that Model-1 has the tightest LP relaxation over all the problem instances, although LP relaxation values are still far from the optimal upper bound.The reason is that the $O\left(n^{4}\right)$ variable formulation is the strongest formulation of the three models. However, this tightness comes at the cost of greatly increased computational time. Using this formulation, no optimal solution found within two hours. We note here that 
Table 1. Results for Models on CAB data sets

\begin{tabular}{|c|c|c|c|c|c|c|c|c|c|c|}
\hline \multirow{2}{*}{ Problem } & \multirow{2}{*}{ Optimal } & \multicolumn{3}{|c|}{ Model-1 } & \multicolumn{3}{|c|}{ Model-2 } & \multicolumn{3}{|c|}{ Model-3 } \\
\hline & & B\&B nodes & $\mathrm{CPU}$ & $\%$ Gap & B\&B nodes & $\mathrm{CPU}$ & $\%$ Gap & B\&B nodes & $\mathrm{CPU}$ & $\%$ Gap \\
\hline 10.2 .1 & 3736.21 & 10,178 & 7200 & 44.83 & 1,312 & 18.48 & 0.0 & 809,124 & 953.86 & 0.0 \\
\hline 10.2 .2 & 2385.71 & 12,182 & 7200 & 22.48 & 3,239 & 171.12 & 0.0 & 343,950 & 2234.15 & 0.0 \\
\hline 10.3 .1 & 2739.41 & 11,557 & 7200 & 35.28 & 7,110 & 303.95 & 0.0 & 191,964 & 420.07 & 0.0 \\
\hline 10.3.2 & 1981.42 & 6,161 & 7200 & 12.11 & 1,591 & 450.86 & 0.0 & 61,080 & 853.54 & 0.0 \\
\hline 15.2.1 & 7037.19 & 17 & 7200 & 78.71 & 207,836 & 6761.28 & 0.0 & $4,071,459$ & 7200.00 & 100 \\
\hline 15.2 .2 & $4174.68^{*}$ & NA & 7200 & NA & 39,321 & 7200.00 & 14.1 & 468,182 & 7200.00 & 100 \\
\hline 15.3 .1 & $4729.81 *$ & NA & 7200 & NA & 85,466 & 7200.00 & 16.98 & 563,138 & 7200.00 & 84.97 \\
\hline 15.3 .2 & $2992.41 *$ & NA & 7200 & NA & 5,919 & 7200.00 & 42.91 & 156,537 & 7200.00 & 100 \\
\hline 15.4.1 & $4105.81 *$ & 22 & 7200 & 67.01 & 23,290 & 7200.00 & 72.97 & 762,886 & 7200.00 & 86.03 \\
\hline 15.4.2 & $3222.86^{*}$ & 135 & 7200 & 37.75 & 1,109 & 7200.00 & 97.31 & 77,582 & 7200.00 & 100 \\
\hline
\end{tabular}

the LP relaxation yielded by Model- 1 is always the longest distance in the data set we considered. However, the LP relaxation value of $\mathrm{O}\left(n^{2}\right)$ variable models are always zero, except in the first problem instance for Model-2.

Lastly, we remark here that the $\mathrm{O}\left(n^{2}\right)$ model with two index vehicle flow formulation has the largest branch and bound tree to be explored compared to other models. We also note here that although Model-3 is based on the most successful formulation in terms of the solution time in the literature for the standard single allocation $p$-hub center problem, the performance of it is weak on the uncapacitated vehicle routing $p$-hub center problem. Clearly, the $\mathrm{O}\left(n^{2} v\right)$ model with three index vehicle flow formulation is superior to Model-1 and Model-3 in terms of the solution quality achieved in reasonable amounts of CPU time.

\section{Conclusions}

In this paper we studied a new hub location problem UVRpHCP. To the best of the authors' knowledge, ours is the first study in the literature that combines vehicle routing and the p-hub center problem. To solve this problem we developed three integer programming models ranging in size form $O\left(n^{2}\right)$ to $O\left(n^{4}\right)$ variables.

We tested the performances of the formulations on the well-known $\mathrm{CAB}$ data set. The numerical results showed the formulation Model-2 with $\mathrm{O}\left(n^{2} v\right)$ variables (where $n$ is the number of nodes and $v$ the number of vehicles) is superior to the other formulations in terms of computational time and solution quality.

Real world problems are, unfortunately, significantly larger than any solved exactly in this paper. Therefore, future work must focus on solving larger problems. This could be achieved by developing new mathematical models that reduce symmetry. For example in all of our formulations renumbering the vehicles leads to equivalent (symmetric) solutions. Reducing such symmetry is likely to have a positive impact but more significant algorithmic improvements are expected to be necessary to obtain exact solutions to medium and large sized instances.

\section{REFERENCES}

Alumur, S. and B. Kara (2008). Network hub location problems: The state of the art. European Journal of Operational Research 190(1), $1-21$.

Campbell, J. (1994). Integer programming formulations of discrete hub location problems. European Journal of Operational Research 72(2), 387 - 405.

Campbell, J. F. and M. O'Kelly (2012). Twenty-five years of hub location research. Transportation Science 46(2), 153-169.

Ernst, A. T., H. Hamacher, H. Jiang, M. Krishnamoorthy, and G. Woeginger (2009). Uncapacitated single and multiple allocation p-hub center problems. Computers \& Operations Research 36(7), 2230-2241.

O'Kelly, M. (1987). A quadratic integer program for the location of interacting hub facilities. European Journal of Operational Research 32(3), 393 - 404.

Rodriguez-Martin, I., J.-J. Salazar-Gonzalez, and H. Yaman (2014). A branch-and-cut algorithm for the hub location and routing problem. Computers \& Operations Research 50(0), $161-174$. 